\title{
1 The feedback between where we go and what we know - Information shapes movement, but movement also impacts information acquisition
}

\author{
3 Orr Spiegel ${ }^{1 *} \&$ Meg Crofoot $^{2,3,4}$ \\ $4 \quad{ }^{1}$ Department of Environmental Science and Policy, University of California, Davis, USA. \\ $5 \quad{ }^{2}$ Department of Anthropology, University of California, Davis, USA. \\ $6 \quad{ }^{3}$ Animal Behavior Graduate Group, University of California, Davis, USA \\ $7 \quad{ }^{4}$ Smithsonian Tropical Research Institute, Balboa, Ancon, Panama, Republic of Panama \\ $8 \quad *$ Corresponding author. Email: orr.spiegel@ huji.ac.il. Mailing Address: 2101 Wickson Hall, One Shields \\ 9 Avenue, Davis, CA 95616, USA.
}

Abstract (100-120)

12 What animals know about their environment is crucial for understanding observed patterns of movement 13 and space-use, but the feedback between information acquisition and movement is rarely considered.

14 Animals can inherit spatial information or obtain it with their senses, via their social environment, or from 15 direct experience. On one hand, this information affects decisions about when, where and how to move.

16 On the other hand, movement itself shapes the information animals acquire due to effects of habitat 17 sampling, speed-accuracy tradeoffs, and cognitive limitations. We suggest that simultaneously considering how the information animals have about their environment affects their movements and how these movements, in turn, shape the information they acquire about their habitat will provide useful insights to the field of movement ecology.

\section{Introduction}

As animals move through their lives in pursuit of necessities like food, water, shelter and mates they create connections between individuals, communities and populations that underlie an array of critical ecological processes [1]. Examples include the forays of foragers which determine the nature and intensity of feeding competition, the excursions of dispersing juveniles which shape the scales of genetic

27 linkage across the landscape and the journeys of long-distance migrants that spread disease across

28 continental boundaries. Together, animals' movement decisions (here, focusing on whole-body

29 locomotion rather than the movements of particular body parts) create fluxes of biomass that shape our 
world at local, regional and global scales [2,3]. A key question about movement at all of these scales is how animals obtain, process and utilize information about their environment to fulfill their goals. Spatial information (e.g., what animals know about where important resources are located in their environment) is central to these decisions about when, where and how to move $[4,5]$. Whereas much of the early research modelling animal movement and space-use assumed unlimited knowledge of the environment [e.g., models based on the ideal free distribution postulate that animals have complete information and no travel costs--6], current research frequently revolves around understanding how locomotor and informational constraints affect animal movement decisions [e.g., how memory and perceptual range shapes foraging behavior--7,8].

The ecological and evolutionary importance of movement, along with technological advances that allow us to track individuals' whereabouts in unprecedented detail have catalyzed an explosion of research to understand the forces driving animal space use [1,2]. Yet, dissecting among these drivers, and determining the fitness-related outcomes of behavioral decisions has proven a challenging task. Observed patterns of individual movement result from interactions and feedbacks between numerous internal [e.g. morphology, age, breeding status, hunger level, personality--9,10,11] and external factors (e.g. resource availability, predation risk, competition) that operate on a broad range of temporal and spatial scales $[1,2,12]$. Even with complete information about an individual's internal state and motivation, as well as its external environment, we can't evaluate the efficiency of its movement strategies without taking into account what the individual knows about its habitat. For instance, a hungry animal may appear to forage in the wrong place, simply since it has a limited perceptual range and is unaware of a nearby prolific patch.

Empirical studies of animal movement ecology rarely take into consideration what information individuals have about their environment [but see 13,14]. In large part, this is due to the logistical difficulties associated with measuring or manipulating an animal's informational state outside of carefully controlled, laboratory settings. Nonetheless, a substantial disconnect also exists between the fields of sensory ecology, ecological cognition, and movement ecology. What animals know about the environment they inhabit remains a black box, which we must crack open if we are to make progress towards understanding the drivers of animal movement decisions, as well as their evolutionary consequences $[15,16]$. Here we argue that considering the feedback process between information and movement can help address this challenge. We suggest that simultaneously considering how the information animals have about their environment affects their movements and how these movements, in turn, shape the information they acquire about their habitat will provide useful insights to the field of movement ecology (Figure 1). While these two links are independently addressed in different disciplines, 
they are rarely considered together. To this end, we briefly summarize how animals obtain spatial information and how it affects their space use. Then we discuss how movements can shape information acquisition and how this feedback can generate testable predictions about animal movement and space use.

\section{How animals obtain spatial information}

Animals may improve their fitness by obtaining information, namely reducing uncertainty about the location and value of important features in their environment, through a few non-mutually exclusive processes. These processes range from a reliance on self- to socially-acquired information, including (i) direct detection via sensory systems, (ii) memory based on personal experience, (iii) inherited information (or 'memory'), and (iv) socially acquired information and cultural traditions [17]. Technically, the latter are also obtained through individuals' sensory modalities but we separate them here since they differ in the source of information. Rather than a binary state - being informed vs. uninformed - information state can often be viewed as a gradient, where individuals integrate information from multiple sources, each with different levels of accuracy and precision.

Self-acquired information is derived from direct detection of the environment within an individual's perpetual range. Animals have evolved a variety of sensory modalities that function at different spatial scales with varying levels of accuracy $[18,19]$. Modalities differ in their probability of detecting cues of interest, as well as in the quality of information they provide once a cue is detected. Variation in quality includes both the precision (the ability to discriminate signal from noise, i.e. to correctly identify a target) and accuracy of the obtained data (the ability to localize the target in space once signal was detected) [Figure 2--5,20]. By combining inputs from multiple sensory modalities, animals may increase the range and quality of the spatial information they can obtain. For example, auditory and/or olfactory cues may beckon animals to areas of high resource density, where modalities with better spatial accuracy (e.g., vision or echolocation) can be used [19]. Further, the multimodality of self-acquired information is important, as it allows animals to use the lack of a cue as a source of information. They can readily abandon areas with no signal and engage in an extensive search in prolific areas, even before encountering the first resource [21].

Animals may also rely on spatial information gained from their own past experiences-i.e. memory. Various theoretical and simulation-based models demonstrate that memory-based foraging strategies outperform memory-free null models across a wide range of ecological conditions $[8,16,22,23]$. Field-based experiments provide evidence that some primates not only use spatial memory, but have the ability to plan efficient foraging routes between multiple target destinations [i.e. use information about 
relative spatial position-24]. Without controlled experiments, it is difficult to determine what role memory plays in shaping animal movement decisions, but repeated visits to particular locations are often viewed as an evidence of memory [22,25], and certain patterns of movement are thought to be tied to the use of memory by foraging animals [e.g., circular nomadism--25,straight-line travel--26]. However, alternate movement strategies can also explain these patterns [15], and increasingly, scientists are taking a model-fitting approach to discern the impact of spatial memory on animal movement decisions $[14,16,23]$.

Spatial information can also be inherited. Genetic encoding and maternal effects may dictate programing, or behavioral rules that influence how individuals respond to cues in their habitat. Since this may contain information that affects spatial movements it can be viewed as 'extended memory' in which individuals 'learn' from the experience of their ancestors. The genetic basis of migration in many species, for instance, includes both the timing and direction of travel routes [27]. Bristle-thighed Curlews (Numenius tahitiensi) present a remarkable example of spatial precision encoded in a migratory gene package - juvenile curlews in their first year of life migrate $4000 \mathrm{~km}$ to a small oceanic island without any guidance from experienced individuals [28]. In other contexts, certain alleles are known to affect foraging and dispersal movements [29]. Essentially, any form of innate habitat preference or cue-taxis behavior that affect movement can be viewed as inherited information. For instance, maternal stress, habitat preferences and nest site selection can influence the tendency of offspring to disperse, as well as their dispersal distances [30-32]. Effectively, such responses to environmental cues use inherited decision rules (or knowledge) acquired though natural selection on the behaviors of one's ancestors, even if they do not represent a learning process.

Finally, animals can obtain information about their habitat from con- and hetero-specifics [17]. By moving as a group, individuals can effectively enhance their detection range beyond their own physiological limitations, and may also improve the quality of information they obtain by integrating among multiple individuals [4,33]. Socially-acquired information may be provided intentionally [e.g. chemical trail-laying by ants--34,the honey bee waggle dance--35], or unintentionally via, for example, local enhancement [36], or scrounging during social foraging [17,37]. The use of roosts as information centers can also facilitate information transfer regarding the location of foraging resources $[38,39]$, as well as enemies and threats [reviewed in 40]. Further, the social transmission of traditions makes it possible for information about past conditions to be transferred across generations $[41,42]$.

How animals integrate information obtained from these different modalities is of great importance for their subsequent space use. Past models have largely considered movement decisions 
based on single cues, and mostly in the context of dispersal and habitat selection. However, a growing body of research in behavioral ecology now focuses on the integration of multiple cues [43-45]. This integration is likely to facilitate our understanding how other types of movement are influenced by the different modalities. Reliance on a diverse set of cues for acquiring spatial information may also impact a species' ability to cope with environmental change [43,45]. Some species will adjust to such changes by altering their movement appropriately, while others, using rules evolved in past environment may face perceptual biases leading to ecological traps where a rapid (human induced) change decouples the cue and the suitability of the habitat they represent [46].

\section{The effect of movements on information acquisition}

In addition to the effect that an animal's information state has on its movement patterns, movement decisions also feedback and shape what an individual knows about its habitat. This feedback includes direct effects on the information available to the animals (i.e., what are the signal and cues the animal experiences en route). It also includes indirect effects on the ability to detect and utilize available information via impacts on one or more of the information acquisition processes outlined above. All organisms have limited cognitive capacity, and attention is always shared among a variety of tasks. For example, moving animals must avoid obstacles while navigating, and simultaneously search for resources and avoid threats [5,20]. Under most conditions, slower travel speeds, straighter trajectories, selection of more hospitable terrain and the use of familiar paths should enhance the attention individuals can allocate to searching their environment [15].

Travel speed is among the most obvious properties of movement that influence information acquisition (Figure 3). The 'speed-accuracy tradeoff' [20] suggests, on one hand, that high travel speeds increase the rate at which moving animals cover ground and encounter targets of interest (especially if these targets are relatively static), thus directly enhancing available information and possibly resource intake rate. On the other hand, faster movements reduce the amount of time an animal is exposed to the relevant cues of each target. One example of this limitation is seen in foraging groups of ring-tailed coatis (Nasua nasua), which are more likely to miss resources when travelling at higher speeds, presumably because they spend a shorter time within the olfactory flume [47]. In addition, 'velocity blur' resulting from high travel speeds also constrains the distance and width of effective visual searches [48]. At the extreme, animals may be essentially blind to potential resources while moving, a phenomenon commonly used to explain widely observed patterns of 'saltatory movement'-intermittent searches where individuals pause during foraging to search for targets $[48,49]$. All else being equal, speed-accuracy tradeoffs should favor intermediate travel speeds when information acquisition is one of the main 
purposes of movement, whereas faster speeds are expected when information gathering is of secondary importance, or the cues of interest are relatively conspicuous [50]. In empirical studies, this tradeoff may only be detectable when direct observation or high resolution tracking is employed, as relocation intervals in the range of seconds to minutes are necessary for estimates of travel speed to reliably represent animals' actual movements [51]. Estimates of travel speed obtained from coarse grained sampling (e.g. hourly fixes) likely represent an integration of various movement modes and thus will be less informative in this context.

Direct effects of other movement properties like tortuosity and directional autocorrelation on the ability to obtain information have been explored less frequently. The effect of these factors might be harder to assess independently of the effect of travel speed per se, since they often co-vary [49]. Path tortuosity, for instance, is often negatively linked to speed due to inertia. On one hand, tortuous movement may facilitate detection due to reduced speed, but on the other hand frequent turning might impair detection due to rapid shifts in the animal's gaze direction and/or the increased attention required to avoid obstacles [50]. Lower straightness reduces travel speed (hence facilitating detection) but may also distract forager attention, amplifying the speed-accuracy tradeoff. We are not familiar with empirical datasets demonstrating these links in free ranging animals. Studies have either accounted for the effect of straightness on speed [e.g., 52], or of speed on detection [e.g., 47,48,53] but not both. Experiments where the detection of food (or a threat) is assessed as a function of movement speed and straightness can further elucidate the interplay between movement properties and information acquisition.

Beyond how animals move, where they travel also affects their familiarity with their environment, modulating their opportunities for acquiring information about their surroundings [54]. Predispersal prospecting behaviors provide dispersing individuals with information about the conditions in their surrounding and can enhance survival and establishment success once they reach destination patches [55-57]. In foraging context, van Overveld \& Matthysen [58,59] manipulated resources in a population of Great Tits (Parus major) and explored the effect of intraspecific variation in exploratory tendency, demonstrating that more exploratory individuals acquired more information on the resource distribution and consequently coped better with temporary removal of resources [7]. The results of this study emphasize that the information gained via exploratory behavior not only mediates access to important resources such as food, but can be a valuable resource, in and of itself. The costs associated with exploratory behavior (increased exposure to risk, time and energy expenditure, and missed-opportunity costs) may be offset by the benefits of increased information [60]. These benefits will be most important in dynamic environments, where resource location and/or quality changes in time and space, and may be minimal in extreme environments where resources are either very static, or highly ephemeral. 
Animals' space use patterns have a direct impact on the environment they experience throughout their lives. Consequently, selective removal of genotypes (e.g., habitat preference as an evolved trait), or parental effects will determine the inherited information passed on to the next generation [30-32]. For instance, as a simple, hypothetical example, an allele enhancing positive photo-taxis in a Planaria flatworm - a maladaptive behavior - will be quickly removed from the population due to the desiccation or predation on individuals that moving into exposed habitat. As a result, subsequent generations will inherit a strong tendency for negative photo-taxis.

Finally, since animals' movement and behavior impact their environment through ecological processes such as consuming and depleting resources, attracting predators and parasites, and physical modifications of their surrounding (e.g., creating trails), moving animals themselves have a direct impact on the information available to them. Informing competitors and mutualist, either intentionally or unintentionally, also alters the social environment and the information available to self and others in a social foraging context [17,37].

\section{Concluding remarks about the signature of information state in movement paths}

Intuitive predictions can be made about the effect than an individual's informational state will have on its patterns of movement and space use. Individuals (or groups) with high quality information about the location of important resources in their habitat are expected to travel in a goal-oriented manner, moving rapidly along straight trajectories as they approach these targets. In contrast, foragers with less complete information, or searching for less conspicuous targets are expected to engage in an extensive search phase, generally characterized by slow, tortuous movements [50]. At larger time scales that include multiple foraging and resting bouts, the movement parameters (i.e. speed, directionality) of informed individuals are expected to be more strongly bi-modal than those of uninformed individuals.

Perhaps less intuitive is the effect of the feedback on the interplay between movement ecology and evolutionary biology. Optimal foraging studies often focus on the effect of movements on short term energetic currencies (e.g., whether one strategy is better than another in maximizing intake rate per unit of time [61]), rather than on performance throughout an individual's entire lifetime track. In contrast, bet hedging theory emphasizes that animals should compromise short term success in order to reduce long term variance in success. In other words, unpredictable environmental changes in time and space should select for animals that invest in (apparently non-optimal on the short term) exploration, in favor of obtaining information on locations of expected future resources [62]. Hence, all else being equal, species (or individuals) that rely more on memory should benefit more from such exploratory sampling, and be willing to sacrifice efficiency in resource exploitation for information gain. Since long-lived species with 
larger brains tend to rely more on memory [16], this leads to the prediction that these species will deviate more from optimal foraging currencies assessed over short terms, rather than displaying highly efficient foraging patterns one might expect to see based on their high levels of encephalization.

In theory, these predictions suggest that comparisons of the movement patterns of sympatric foragers can yield important insights into the relative quality of the information they possess about their habitat. For instance, all else being equal (e.g., their resource distribution and locomotion capacities) individuals with longer detection distances or better spatial memory are predicted to have longer approach phases, characterized by straight fast travel, as they near important movement goals $[15,26]$. In practice, however, the past experience of study animals, their sensory capacities, and the influence of conspecifics on their behavior are generally unknown in most empirical movement studies, making it difficult, if not impossible, to disentangle the relative importance of the different sources of information in shaping their movement decisions. Since movements consistent with one explanation might as well result from other processes, inferring the underlying mechanism from apparent movement patterns is problematic without convincing evidence against alternative explanations of observed patterns $[15,16]$. For example, an increase in travel speed as individuals approach important movement targets (but before the target is close enough to be detected) is often interpreted as an evidence for the use of spatial memory in primates $[63,64]$. However, this higher travel speed may also reflect other mechanisms such as increasing certainty about the group destination or competitive foraging dynamics. We believe that excluding alternative explanations may only be achievable by either collecting complementary, non-movement data on individuals' behavior or the relative quality of the sites they visit $[25,57,64]$, or conducting field-based experiments [15,24,47]. For instance, using experimental food platforms, Janson and DiBitetti [53] were able to empirically estimate capuchin monkeys' resource detection distance under field conditions, and thus disentangle the role of memory vs. perception in groups' movement decisions [24]. While logistically challenging, we argue that such an experimental approach can provide invaluable insights on the mechanisms that shape the feedback between information and movement in free-ranging animals.

\section{Acknowledgments:}

We are grateful to T. Avgar and an anonymous reviewer for their valuable feedback on this manuscript; and to A. Sih and A. Kacelnik for their invitation to contribute to this special feature. OS was supported by the NSF grant (DEB-1456730) to A. Sih. MCC acknowledges support from the NSF (BCS-1440755, IOS-1250895 and III-1514174). 
References (see below for annotated references):

1. Kays R, Crofoot MC, Jetz W, Wikelski M: Terrestrial animal tracking as an eye on life and planet. Science 2015, 348.

2. Holyoak M, Casagrandi R, Nathan R, Revilla E, Spiegel O: Trends and missing parts in the study of movement ecology. Proceedings of the National Academy of Sciences 2008, 105:19060-19065.

3. Nathan R, Getz W, Revilla E, Holyoak M, Kadmon R, Saltz D, Smouse P: A movement ecology paradigm for unifying organismal movement research. Proceedings of the National Academy of Sciences of the United States of America 2008, 105:19052 - 19059.

4. Dall SR, Giraldeau L-A, Olsson O, McNamara JM, Stephens DW: Information and its use by animals in evolutionary ecology. Trends in Ecology \& Evolution 2005, 20:187-193.

5. Schmidt KA, Dall SR, Van Gils JA: The ecology of information: an overview on the ecological significance of making informed decisions. Oikos 2010, 119:304-316.

6. Fretwell SD, Calver JS: On territorial behavior and other factors influencing habitat distribution in birds. Acta Biotheoretica 1969, 19:37-44.

7. Fronhofer EA, Hovestadt T, Poethke HJ: From random walks to informed movement. Oikos 2013, 122:857-866.

8. Gautestad AO: ANIMAL SPACE USE: Memory Effects, Scaling Complexity, and Biophysical Model Coherence: Dog Ear Publishing; 2015.

9. Dingle H: Migration: the biology of life on the move: Oxford University Press, USA; 2014.

10. Spiegel O, Harel R, Getz WM, Nathan R: Mixed strategies of griffon vultures'(Gyps fulvus) response to food deprivation lead to a hump-shaped movement pattern. Movement Ecology 2013, 1:112.

11. Spiegel O, Leu ST, Sih A, Godfrey SS, Bull CM: When the going gets tough: behavioural typedependent space use in the sleepy lizard changes as the season dries. Proc. R. Soc. B 2015, 282:20151768.

12. Nathan R, Getz WM, Revilla E, Holyoak M, Kadmon R, Saltz D, Smouse PE: A movement ecology paradigm for unifying organismal movement research. Proceedings of the National Academy of Sciences of the United States of America 2008, 105:19052-19059.

13. Strandburg-Peshkin A, Twomey CR, Bode NWF, Kao AB, Katz Y, loannou CC, Rosenthal SB, Torney CJ, Wu HS, Levin $S A$, et al.: Visual sensory networks and effective information transfer in animal groups. Current biology : CB 2013, 23:R709-R711.

14. Avgar T, Baker JA, Brown GS, Hagens JS, Kittle AM, Mallon EE, McGreer MT, Mosser A, Newmaster SG, Patterson BR: Space-use behaviour of woodland caribou based on a cognitive movement model. Journal of Animal Ecology 2015, 84:1059-1070.

15. Janson $\mathrm{CH}$, Byrne R: What wild primates know about resources: opening up the black box. Animal Cognition 2007, 10:357-367.

16. Fagan WF, Lewis MA, Auger-Méthé M, Avgar T, Benhamou S, Breed G, LaDage L, Schlägel UE, Tang Ww, Papastamatiou YP: Spatial memory and animal movement. Ecology Letters 2013, 16:13161329.

17. Danchin É, Giraldeau L-A, Valone TJ, Wagner RH: Public information: from nosy neighbors to cultural evolution. Science 2004, 305:487-491.

18. Stevens M: Sensory ecology, behaviour, and evolution: OUP Oxford; 2013.

19. Nevitt GA: Sensory ecology on the high seas: the odor world of the procellariiform seabirds. Journal of Experimental Biology 2008, 211:1706-1713.

20. Chittka L, Skorupski P, Raine NE: Speed-accuracy tradeoffs in animal decision making. Trends in Ecology \& Evolution 2009, 24:400-407. 
21. Hein AM, McKinley SA: Sensing and decision-making in random search. Proceedings of the National Academy of Sciences 2012, 109:12070-12074.

22. Bracis C, Gurarie E, Van Moorter B, Goodwin RA: Memory effects on movement behavior in animal foraging. PLOS ONE 2015, 10:e0136057.

23. Schlägel UE, Lewis MA: Detecting effects of spatial memory and dynamic information on animal movement decisions. Methods in Ecology and Evolution 2014, 5:1236-1246.

24. Janson $\mathrm{CH}$ : Experimental evidence for spatial memory in foraging wild capuchin monkeys, Cebus apella. Animal Behaviour 1998, 55:1229-1243.

25. Merkle J, Fortin D, Morales J: A memory-based foraging tactic reveals an adaptive mechanism for restricted space use. Ecology Letters 2014, 17:924-931.

26. Polansky L, Kilian W, Wittemyer G: Elucidating the significance of spatial memory on movement decisions by African savannah elephants using state-space models. Proceedings of the Royal Society of London B: Biological Sciences 2015, 282:20143042.

27. Liedvogel M, Åkesson S, Bensch S: The genetics of migration on the move. Trends in Ecology \& Evolution 2011, 26:561-569.

28. Marks JS, Redmond RL: Migration of Bristle-thighed Curlews on Laysan Island: timing, behavior and estimated flight range. Condor 1994:316-330.

29. Edelsparre $\mathrm{AH}$, Vesterberg $\mathrm{A}$, Lim JH, Anwari M, Fitzpatrick MJ: Alleles underlying larval foraging behaviour influence adult dispersal in nature. Ecology Letters 2014, 17:333-339.

30. Bitume $E$, Bonte $D$, Ronce $O$, Olivieri I, Nieberding C: Dispersal distance is influenced by parental and grand-parental density. Proceedings of the Royal Society of London B: Biological Sciences 2014, 281:20141061.

31. Duckworth RA, Belloni V, Anderson SR: Cycles of species replacement emerge from locally induced maternal effects on offspring behavior in a passerine bird. Science 2015, 347:875-877.

32. Clobert J, Baguette M, Benton TG, Bullock JM, Ducatez S: Dispersal ecology and evolution: OUP Oxford; 2012.

33. Codling E, Pitchford J, Simpson S: Group navigation and the "many-wrongs principle" in models of animal movement. Ecology 2007, 88:1864-1870.

34. Wilson EO: Chemical communication among workers of the fire ant Solenopsis saevissima (Fr. Smith) 1. The organization of mass-foraging. Animal Behaviour 1962, 10:134-147.

35. Von Frisch K: The dance language and orientation of bees. 1967.

36. Buckley NJ: Food finding and the influence of information, local enhancement, and communal roosting on foraging success of North American vultures. The Auk 1996:473-488.

37. Giraldeau LA, Caraco T: Social Foraging Theory. Princeton, New Jersey: Princeton University Press; 2000.

38. Ward P, Zahavi A: The importance of certain assemblages of birds as "information-centres" for food-finding. IBIS 1973, 115:517-534.

39. O'Mara MT, Dechmann DK, Page RA: Frugivorous bats evaluate the quality of social information when choosing novel foods. Behavioral Ecology 2014:aru120.

40. Evans JC, Votier SC, Dall SR: Information use in colonial living. Biological Reviews 2015.

41. Fishlock V, Caldwell C, Lee PC: Elephant resource-use traditions. Animal Cognition 2016, 19:429-433.

42. Brent L, Franks DW, Foster EA, Balcomb KC, Cant MA, Croft DP: Ecological knowledge, leadership, and the evolution of menopause in killer whales. Current Biology 2015, 25:746-750.

43. Sih A, Ferrari MC, Harris DJ: Evolution and behavioural responses to human-induced rapid environmental change. Evolutionary Applications 2011, 4:367-387.

44. Fahrig L: Non-optimal animal movement in human-altered landscapes. Functional Ecology 2007, 21:1003-1015. 
45. Sih A, Trimmer PC, Ehlman SM: A conceptual framework for understanding behavioral responses to HIREC. Current Opinion in Behavioral Sciences 2016.

46. Gilroy JJ, Sutherland WJ: Beyond ecological traps: perceptual errors and undervalued resources. Trends in Ecology \& Evolution 2007, 22:351-356.

47. Hirsch BT: Tradeoff Between Travel Speed and Olfactory Food Detection in Ring-Tailed Coatis (Nasua nasua). Ethology 2010, 116:671-679.

48. Kramer DL, McLaughlin RL: The behavioral ecology of intermittent locomotion. American Zoologist 2001, 41:137-153.

49. Campos D, Méndez V, Bartumeus F: Optimal intermittence in search strategies under speedselective target detection. Physical Review Letters 2012, 108:028102.

50. Spiegel O, Getz WM, Nathan R: Factors influencing foraging search efficiency: why do scarce lappetfaced vultures outperform ubiquitous white-backed vultures? The American Naturalist 2013, 181:E102-E115.

51. Marcus Rowcliffe J, Carbone C, Kays R, Kranstauber B, Jansen PA: Bias in estimating animal travel distance: the effect of sampling frequency. Methods in Ecology and Evolution 2012.

52. Grodzinski U, Spiegel O, Korine C, Holderied MW: Context-dependent flight speed: evidence for energetically optimal flight speed in the bat Pipistrellus kuhlii? Journal of Animal Ecology 2009, 78:540-548.

53. Janson CH, DiBitetti MS: Experimental analysis of food detection in capuchin monkeys: effects of distance, travel speed, and resource size. Behavioral Ecology and Sociobiology 1997, 41:17-24.

54. Trimmer PC, Houston Al: An evolutionary perspective on information processing. Topics in cognitive science 2014, 6:312-330.

55. Bocedi G, Heinonen J, Travis JM: Uncertainty and the role of information acquisition in the evolution of context-dependent emigration. The American Naturalist 2012, 179:606-620.

56. Delgado MdM, Bartoń K, Bonte D, Travis J: Prospecting and dispersal: their eco-evolutionary dynamics and implications for population patterns. Proceedings of the Royal Society of London B: Biological Sciences 2014, 281:20132851.

57. Spiegel O, Harel R, Centeno-Cuadros A, Hatzofe O, Getz WM, Nathan R: Moving beyond Curve Fitting: Using Complementary Data to Assess Alternative Explanations for Long Movements of Three Vulture Species. The American Naturalist 2015, 185:E44-E54.

58. van Overveld T, Matthysen E: Personality predicts spatial responses to food manipulations in freeranging great tits (Parus major). Biology Letters 2010, 6:187-190.

59. van Overveld T, Matthysen E: Personality and information gathering in free-ranging great tits. PLoS ONE 2013, 8:e54199.

60. Berger-Tal O, Avgar T: The glass is half-full: overestimating the quality of a novel environment is advantageous. PLoS ONE 2012, 7:e34578.

61. Stephens DW, Krebs JR: Foraging Theory. Princeton: Princeton University Press; 1986.

62. Simons AM: Modes of response to environmental change and the elusive empirical evidence for bet hedging. Proceedings of the Royal Society of London B: Biological Sciences 2011:rspb20110176.

63. Pochron ST: Can concurrent speed and directness of travel indicate purposeful encounter in the yellow baboons (Papio hamadryas cynocephalus) of Ruaha National Park, Tanzania? International Journal of Primatology 2001, 22:773-785.

64. Janmaat KR, Byrne RW, Zuberbühler K: Evidence for a spatial memory of fruiting states of rainforest trees in wild mangabeys. Animal Behaviour 2006, 72:797-807. 


\section{References of special interest (*)}

- Merkle et al 2014. This study explores inter patch movements of free ranging female bison and shows that they remember the location and quality of meadows, and reduced uncertainty in foraging success by returning to previously prolific sites, especially if their recent visits were to lower quality meadows.

- O'Mara et al 2014. This study combines lab experiments with field observations to demonstrate that bats pay attention to the quality of the social information. Bats learn from roost-mates' odors about novel food types. Further, they discriminate between odors on breath vs. fur. The latter cue indicates consumption by the roost-mate, allowing the inexperienced individual to select the most informative cue on the quality of novel food.

- Kays, et al 2015. A recent overview on animal tracking in terrestrial systems, covering the main questions currently addressed in movement ecology, some of the common methods used in the field and the potential challenges.

- Spiegel et al 2015. This papers explores a prospecting behavior of adult vultures where Individuals engage in long, circular forays. By adding behavioral identification from accelerometer data to the GPS positional data, the authors show these forays cannot be explained by optimal foraging criteria and call for contrasting movement data with complementary datasets.

\section{References of outstanding interest $(* *)$}

J Fronhofer et al. 2013. An elegant modeling approach demonstrating how space-use patterns that resemble commonly-used random walks simulation can be obtained from a more realistic approach with a mechanistic model that is based on information use and memory. Their analysis shows how informed foraging outperforms other strategies.

fo Fagan et al. 2013. A review in the linkages between memory and animal movement. They explore the adaptive costs and benefits of memory as outlined in psychology and neuroscience, and describe how emerging movement pattern can relate to memory, and how experiments can distinguish this effect from other alternatives.

J Avgar et al. 2015. This paper expands on a previously developed model of the cognitive processes that affect animal space use. The model evaluates the contributions of perception, memory and decision making to animal movement patterns, and the authors use it to a dataset of GPS tracks of woodland caribou with respect to food availability, predator abundance (wolves), and apparent competitors (moose). 
J van Overveld \& Matthysen 2013. This study continues the analysis of a field experiment that included temporary removal of feeding stations used by great tits. They show that consistent intraspecific variation in exploratory behavior affected how birds obtained spatial information and in their tendency to revisit empty feeders, consequently affecting their style of coping with environmental change.

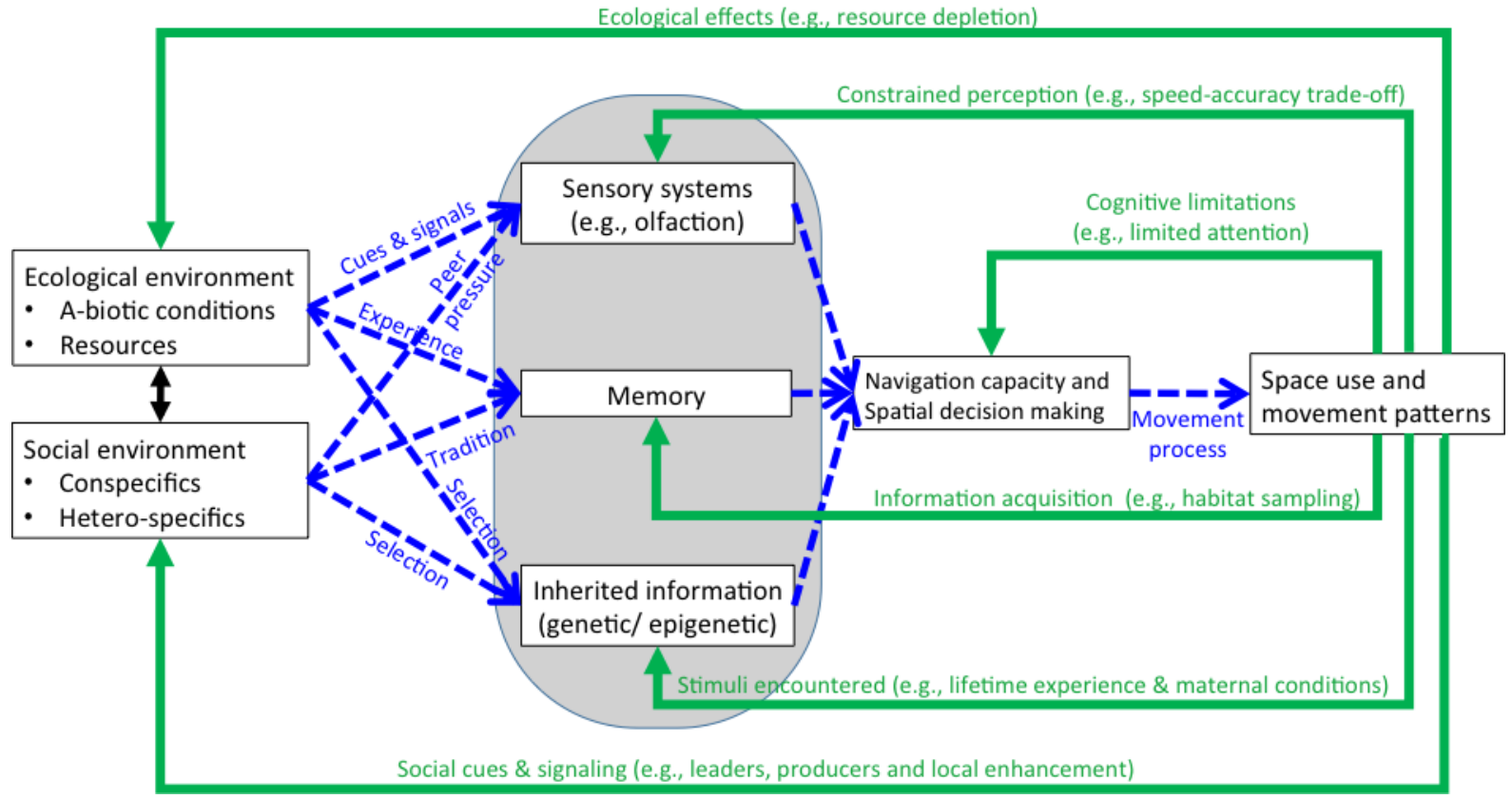

Figure 1. Animals obtain information about their ecological and social environments through a few non-mutually exclusive processes (grey oval). This information is integrated into decisions that translate to movement patterns (e.g., a directed and fast movement towards a known target). Movement patterns, in turn, affect further information acquisition by changing the signals and cues animals experience, as well as their ability to acquire and process this information. For instance, moving faster shortens the time window for detecting a resource, constrains perceptual range and reduces attention that can be devoted for search. Blue dashed arrows illustrate the effects that information has on movement while the green arrows reflect the feedback of movement on information acquisition. 


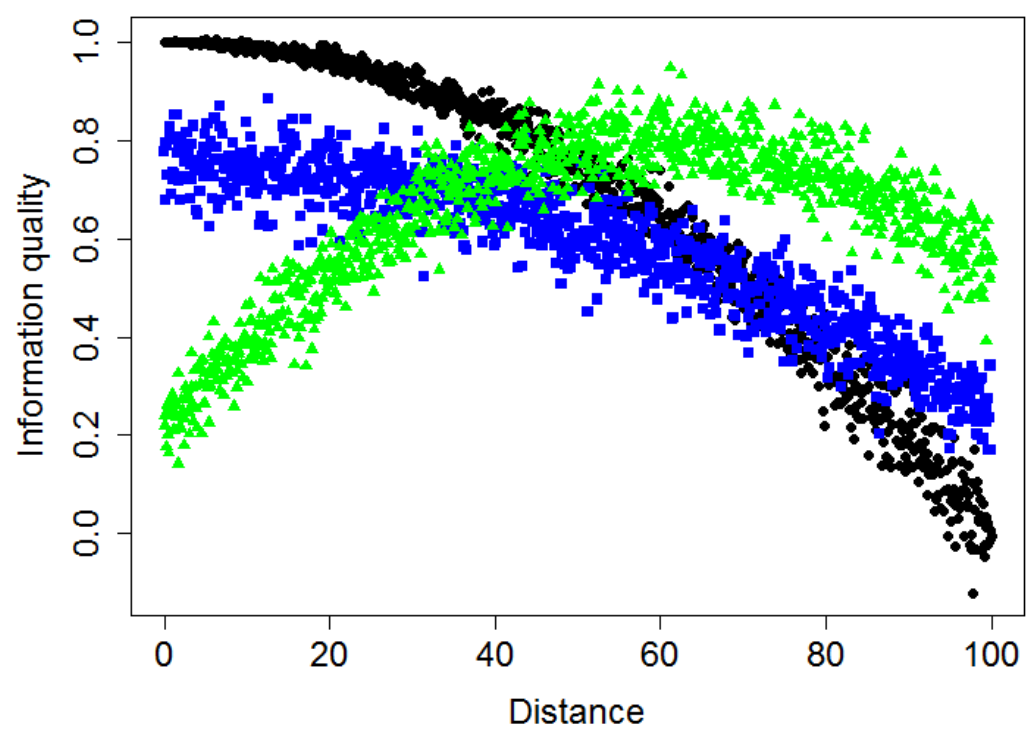

443

444 Figure 2. Hypothetical detection functions for three sensory modalities with information quality as a function 445 of distance from the target: visual (black circles), olfactory (green triangles) and auditory (blue squares). Note 446 that modalities differ in their response to distance both in the decay (precision) and in the spread (accuracy). 


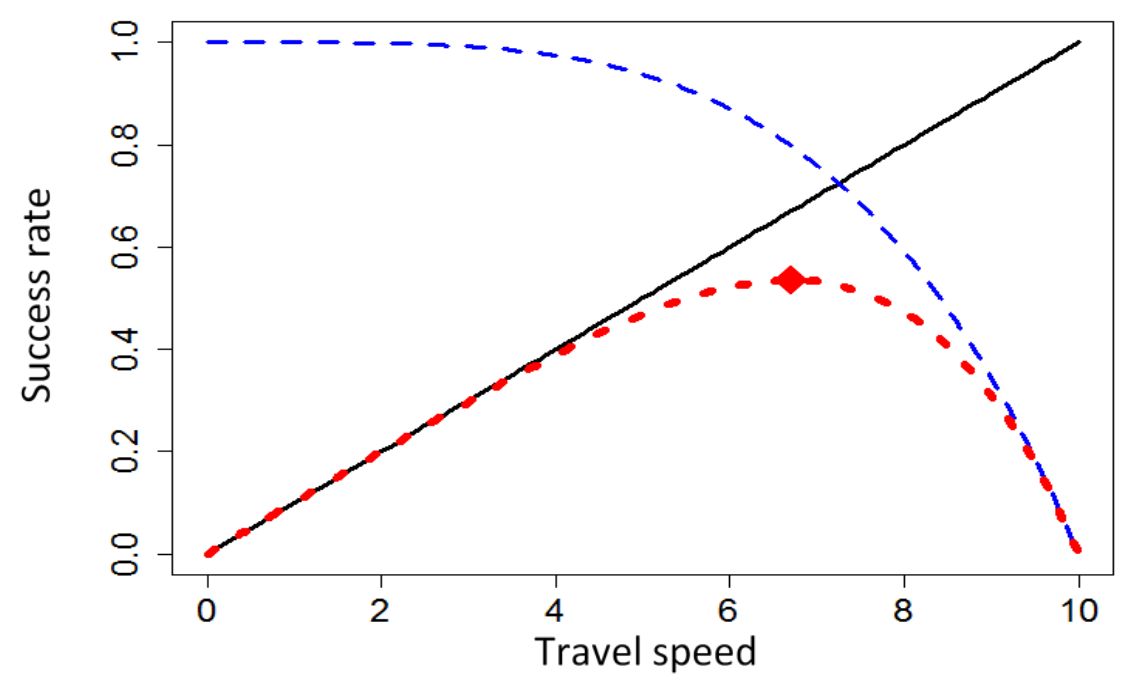

Figure 3. The effect of travel speed on foraging success. For relatively static resources encounter rate

450 (black solid line) depends on distance travelled and grows linearly with speed. Probability of detection (blue

451 dashed line) declines non-linearly with speed due to reduced perception and limited attention at high travel

452 speeds. The overall finding success (red dotted line) is the product of the two, with optimal search speed

453 marked with a diamond. All parameters in this hypothetical example are scaled. 


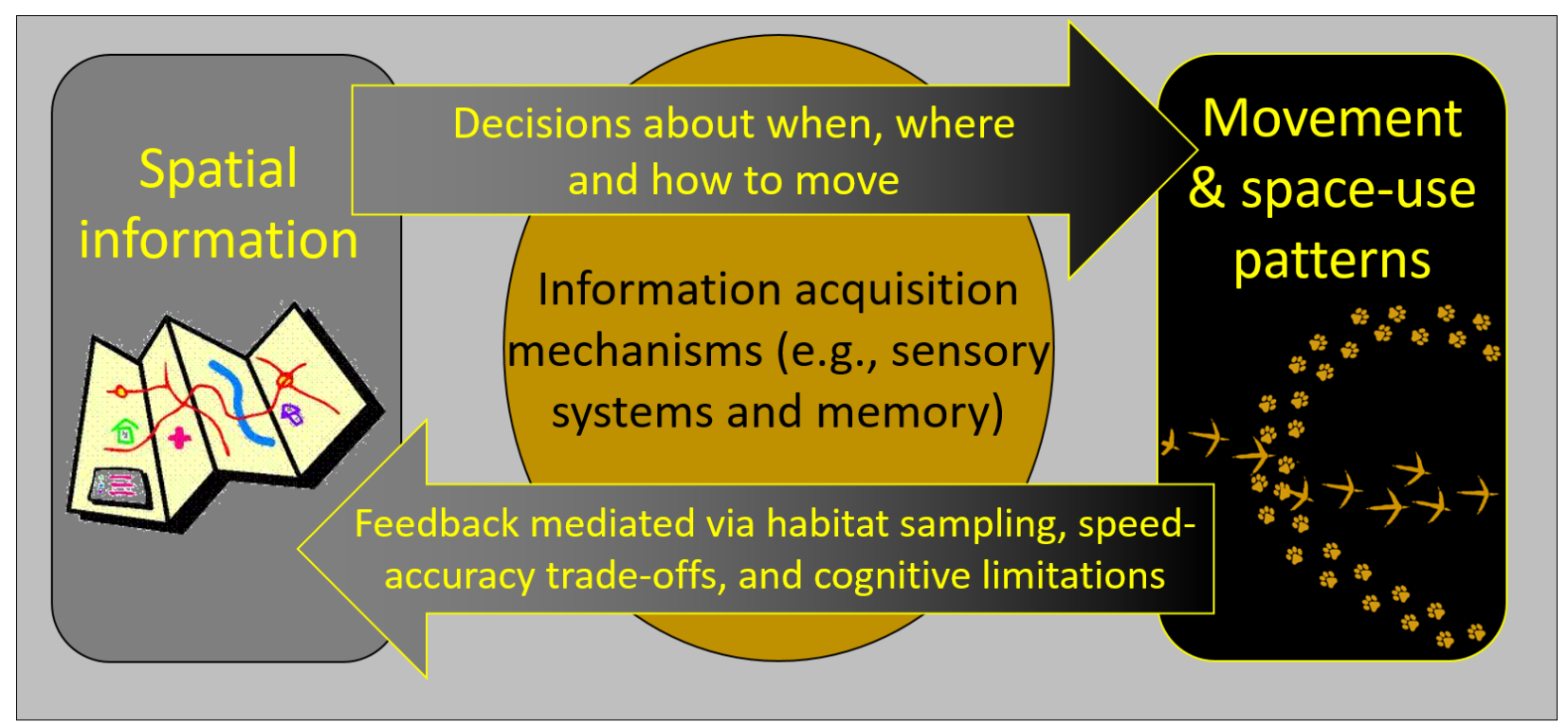

